\title{
Utilization of breast cancer screening with magnetic resonance imaging in community practice
}

\author{
Deirdre A. Hill, Ph.D. ${ }^{1,2}$, Jennifer S. Haas, M.D. ${ }^{3}$, Robert Wellman, M.S. ${ }^{4}$, Rebecca A. Hubbard, Ph.D ${ }^{5}$, \\ Christoph I. Lee, M.D.6.7, Jennifer Alford-Teaster, M.P. H, ${ }^{8,9}$, Karen J. Wernli, Ph.D. ${ }^{4}$, \\ Louise M. Henderson, Ph.D. ${ }^{10}$, Natasha K. Stout, Ph.D. ${ }^{17}$, Anna N. A. Tosteson, Sc.D ${ }^{12}$, \\ Karla Kerlikowske, M.D. ${ }^{13}$, and Tracy Onega, Ph.D. $8,9,12$
}

\begin{abstract}
'Department of Internal Medicine and Comprehensive Cancer Center, University of New Mexico School of Medicine, Albuquerque, NM, USA; ${ }^{2}$ Department of Internal Medicine, University of New Mexico School of Medicine, Albuquerque, NM, USA; ${ }^{3}$ Division of General Internal Medicine and Primary Care, Brigham and Women's Hospital, Boston, MA, USA; ${ }^{4}$ Group Health Research Institute, Seattle, WA, USA; 5 Department of Biostatistics, Epidemiology \& Informatics, Perelman School of Medicine, University of Pennsylvania, Philadelphia, PA, USA; ${ }^{6}$ Department of Radiology, University of Washington School of Medicine, Seattle, WA, USA; ${ }^{7}$ Department of Health Services, University of Washington School of Public Health, Seattle, WA, USA; ${ }^{8}$ Departments of Biomedical Data Science and Epidemiology, Geisel School of Medicine at Dartmouth, Lebanon, $\mathrm{NH}$, USA; ${ }^{9}$ Norris Cotton Cancer Center, Geisel School of Medicine at Dartmouth, Lebanon, NH, USA; ${ }^{10}$ Department of Radiology, University of North Carolina, Chapel Hill, NC, USA; " Department of Population Medicine, Harvard Medical School and Harvard Pilgrim Health Care Institute, Boston, MA, USA; ${ }^{2}$ Department of Medicine, The Dartmouth Institute for Health Policy and Clinical Management and Norris Cotton Cancer Center, Geisel School of Medicine at Dartmouth, Lebanon, NH, USA; ${ }^{13}$ Departments of Medicine and Epidemiology/Biostatistics, University of California, San Francisco, CA, USA.
\end{abstract}

BACKGROUND: Breast cancer screening with magnetic resonance imaging (MRI) may be a useful adjunct to screening mammography in high-risk women, but MRI uptake may be increasing rapidly among low- and average-risk women for whom benefits are unestablished. Comparatively little is known about use of screening MRI in community practice.

OBJECTIVE: To assess relative utilization of MRI among women who do and do not meet professional society guidelines for supplemental screening, and describe utilization according to breast cancer risk indications.

DESIGN: Prospective cohort study conducted between 2007 and 2014.

PARTICIPANTS: In five regional imaging registries participating in the Breast Cancer Surveillance Consortium (BCSC), 348,955 women received a screening mammogram, of whom 1499 underwent screening MRI.

MAIN MEASURES: Lifetime breast cancer risk $(<20 \%$ or $\geq 20 \%$ ) estimated by family history of two or more firstdegree relatives, and Gail model risk estimates. Breast Imaging Reporting and Data System breast density and benign breast diseases also were assessed. Relative risks (RR) for undergoing screening MRI were estimated using Poisson regression.

KEY RESULTS: Among women with < 20\% lifetime risk, which does not meet professional guidelines for supplementary MRI screening, and no first-degree breast cancer family history, screening MRI utilization was elevated among those with extremely dense breasts [RR 2.2; 95\% confidence interval (CI) 1.7-2.8] relative to those with scattered fibroglandular densities and among women with atypia (RR 7.4; 95\% CI 3.9-14.3.) or lobular carcinoma in situ (RR 33.1; 95\% CI 18.0-60.9) relative to women

Received February 28, 2017

Revised September 29, 2017

Accepted October 31, 2017

Published online December 6, 2017 with non-proliferative disease. Approximately $82.9 \%$ (95\% CI 80.8\%-84.7\%) of screening MRIs occurred among women who did not meet professional guidelines and 35.5\% (95\% CI 33.1-37.9\%) among women considered at low-to-average breast cancer risk.

CONCLUSION: Utilization of screening MRI in community settings is not consistent with current professional guidelines and the goal of delivery of high-value care.

KEY WORDS: breast neoplasms; magnetic resonance imaging; guideline adherence; early detection of cancer; risk factors.

J Gen Intern Med 33(3):275-83

DOI: $10.1007 /$ s11606-017-4224-6

(C) Society of General Internal Medicine 2017

\section{INTRODUCTION}

Magnetic resonance imaging (MRI) has become much more widely utilized for breast cancer screening ${ }^{1,2}$ since guidelines for MRI use in high-risk women were first issued in 2007. MRI is currently recommended for breast cancer screening among women with $20 \%$ or greater lifetime breast cancer risk by the American Cancer Society, the American College of Radiology, and the Society of Breast Imaging. ${ }^{3}$ The American College of Physicians advises against MRI screening for average-risk women. ${ }^{4}$ While much of the data regarding potential harms and benefits of screening MRI are derived from studies in high-risk populations, ${ }^{5-9}$ evidence is accumulating that screening MRI is diffusing to average-risk women, ${ }^{1,2}$ for whom the benefit/harm ratio is unknown, and possibly lower.

Among women with a high breast cancer risk, annual screening with MRI in conjunction with mammography has led to increased cancer detection rates compared with mammography alone. ${ }^{5-9}$ In high-risk women, cancers detected by MRI are more likely to be earlier stage and node negative than 
those detected by mammography. ${ }^{5,}{ }^{10}$ However, providers do not always recognize when women meet criteria for high breast cancer risk and should be referred for related services. ${ }^{11}$ Although benefits of MRI screening of high-risk women are often accompanied by higher false-positive rates than mammography screening 5, 7, 12 and direct evidence of decreased mortality with MRI use is lacking, low rates of supplemental MRI screening of women with $20 \%$ or greater lifetime risk could be considered a missed opportunity to utilize a technology that demonstrates promise for early detection.

Despite lack of data regarding benefit, results of several studies suggest that MRI screening is diffusing to lower risk women not covered by professional society recommendations. ${ }^{1,2}$ Among women with less than $20 \%$ lifetime risk, performance of screening breast MRI, including cancer detection rates, false-positive rates, and recalls for biopsy and other diagnostic tests, has not been determined. In decision analytic models, screening MRI is not cost-effective unless utilized in extremely high-risk populations. ${ }^{13,14}$ In the absence of data on the balance of benefits and harms, MRI screening among women with less than $20 \%$ lifetime risk might be considered unwarranted use of the technology. Relatively little is known about MRI utilization patterns in community practice.

Professional guidelines for screening MRI recommend that lifetime risk be determined by prediction models that use family history or high-risk mutation status as primary risk determinants. In models of lifetime risk based on family history, such as the Claus model, the International Breast Cancer Intervention Model, or the Breast and Ovarian Analysis of Disease Incidence and Carrier Estimation Algorithm, ${ }^{15-}$ ${ }^{17}$ generally, a woman with two first-degree relatives with breast cancer has at least $20 \%$ lifetime risk and meets the threshold for annual MRI screening.

We sought to determine the utilization of supplemental screening with breast MRI among 348,955 women receiving mammography screening in the Breast Cancer Surveillance Consortium (BCSC). We examined MRI screening according to established breast cancer risk factors, using two or more first-degree relatives with breast cancer as an indicator of $20 \%$ or greater lifetime breast cancer risk.

\section{METHODS}

\section{Study Setting and Data Sources}

The study was conducted in five regional registries of the BCSC, a breast imaging network that collects risk factor, imaging, and pathology information as well as cancer diagnoses and vital statistics data for women undergoing breast cancer screening. Cancer diagnosis information is obtained from linkage to state tumor registries or to National Cancer Institute (NCI)-funded Surveillance, Epidemiology, and End Results (SEER) registries. Women who received mammograms during 2007-2014 at imaging facilities participating in the BCSC were eligible for this study. Data for this analysis were collected from registries in California (San Francisco Mammography Registry, SFMR), Vermont (Vermont Breast Cancer Surveillance System, VBCSS), Washington State (Group Health Breast Cancer Surveillance Registry, GH), North Carolina (Carolina Mammography Registry, CMR), and New Hampshire (New Hampshire Mammography Network, NHMN). ${ }^{18}$ Each registry obtained Institutional Review Board approval for either active or passive consent processes or a waiver of consent to enroll participants, link data, and perform analysis. All procedures were Health Insurance Portability and Accountability Act (HIPAA) compliant. All registries and the Statistical Coordinating Center obtained a Federal Certificate of Confidentiality and other protections for the identities of women, physicians, and facilities who are subjects of this research.

\section{Measures, Definitions, and Outcomes}

All women aged 35-69 years without a personal history of ductal carcinoma in situ, invasive breast cancer, or breast augmentation who received a screening mammogram at a BCSC facility between 2007 and 15 months prior to the end of follow-up (NHMN December 2011; GH September 2013; SFMR and CMR December 2013; VBCSS September 2014) were eligible. A screening mammogram was defined as a bilateral mammogram with indication of "screening" recorded by the radiologist or technologist, which was not preceded by a mammography examination within the previous 9 months. A minimum of 15 months of follow-up was required to allow capture of receipt of screening MRI, our primary outcome. We restricted analyses to women screened at facilities that reported at least five MRI examinations during the study period to insure that MRI was available to attendees.

Demographic and risk factor information was collected using self-administered forms at the time of mammography. Women reported age, race/ethnicity, highest level of educational attainment, and first-degree family history of breast cancer. Prior biopsy results obtained from pathology reports were categorized as: non-proliferative disease, which included unknown; proliferative disease without atypia, atypical hyperplasia, and lobular carcinoma in situ (LCIS). ${ }^{19}$ Breast mammographic density obtained from radiologist assessment of mammograms was classified according to American College of Radiology Breast Imaging-Reporting and Data System (BI-RADS) criteria as almost entirely fat, scattered fibroglandular tissue, heterogeneously dense, and extremely dense. ${ }^{20}$

The primary exposure of interest was $20 \%$ or greater lifetime breast cancer risk as defined by family history of two or more first-degree relatives with breast cancer, which usually exceeds the $20 \%$ threshold. ${ }^{15-17}$ We also calculated lifetime risk according to the NCI Breast Cancer Risk Assessment Tool, also known as the Gail model. ${ }^{21}$ Standard breast cancer risk factors included in the Gail model are current age, age at menarche and first live birth, number of first-degree relatives 
with breast cancer, number of breast biopsies, and history of breast atypia.

Women were considered to have received supplemental screening MRI if an MRI with clinical indication "screening (asymptomatic)" was performed within 15 months before or 15 months following the screening mammogram.

\section{Statistical Analysis}

We compared the probability of being screened with MRI by patient characteristics, estimating adjusted relative risks (RR) of receiving a screening MRI and 95\% confidence limits using modified Poisson regression estimated via generalized estimating equations. We used robust standard errors to account for violation of the Poisson mean-variance relationship. ${ }^{22}$ To allow for the additional opportunity to obtain a screening MRI among women who received more than one mammogram during the study period, the total number of mammography examinations observed for each woman was included as an offset in the Poisson model. All RR estimates were adjusted for age at first examination during the study period and for BCSC registry. Analyses were stratified by high risk $(\geq 20 \%$ lifetime risk) vs. average/low-risk $(<20 \%$ lifetime risk $)$ and age at screening examination ( $<50$ years, $\geq 50$ years). As the $\mathrm{BCSC}$ is an open cohort, women receiving mammograms at a BCSC facility may have received screening MRI at a facility outside BCSC catchment. Thus, we report RRs for patient characteristics associated with MRI use, which are unbiased under imperfect outcome ascertainment. ${ }^{23}$ All analyses were performed using SAS software v9.4 (Cary, NC).

\section{RESULTS}

Women screened with mammography included in the analysis $(n=348,955)$ underwent 1499 screening MRIs and were predominantly non-Hispanic white $(69.9 \%)$ and college educated $(53.3 \%)$ (Table 1). More than 43,000 of these women $(12.5 \%)$ reported a first-degree family history of breast cancer. Approximately $1.4 \%$ of included women had a lifetime breast cancer risk of $20 \%$ or more according to the Gail risk model.

Women who reported either no or one first-degree relative with breast cancer, and who were therefore not eligible for screening MRI according to published guidelines, were $98.8 \%$ of included women ( $n=344,858$; 95\% CI 98.8-98.9\%) (Table 2). Among these women, those with established breast cancer risk factors were much more likely to undergo screening MRI. Women with one first-degree relative with breast cancer had a greater than eight-fold increased risk of undergoing a screening MRI compared with women with no affected first-degree relatives; an estimated $47.4 \%$ (710/1499; 95\% CI 44.8-49.9\%) of all screening MRIs was received by these women.

Among women who did not have a first-degree family history, we investigated other risk factors that might have prompted MRI screening. Women who reported a prior biopsy were 4.0 times more likely to undergo supplemental MRI than
Table 1 Characteristics of Women Who Received Breast Cancer Screening in the Breast Cancer Surveillance Consortium $(n=348,955)$

\begin{tabular}{|c|c|c|}
\hline Characteristic & $\mathbf{N}$ & $\%$ \\
\hline \multicolumn{3}{|l|}{ Age at first examination (years) } \\
\hline $35-39$ & 16,141 & 4.6 \\
\hline $40-49$ & 129,122 & 37.0 \\
\hline $50-59$ & 123,249 & 35.3 \\
\hline $60-69$ & 80,443 & 23.1 \\
\hline \multicolumn{3}{|l|}{ Race/ethnicity } \\
\hline Non-Hispanic White & 222,951 & 69.9 \\
\hline Non-Hispanic Black & 23,155 & 7.3 \\
\hline Hispanic & 20,453 & 6.4 \\
\hline Other & 52,368 & 16.4 \\
\hline Missing & 30,028 & \\
\hline \multicolumn{3}{|l|}{ Highest level of education } \\
\hline HS grad/GED or less & 62,092 & 21.2 \\
\hline Some college/technical & 74,868 & 25.5 \\
\hline College grad or higher & 156,586 & 53.3 \\
\hline Missing & 55,409 & \\
\hline \multicolumn{3}{|l|}{ First-degree family history of breast cancer } \\
\hline No & 305,503 & 87.6 \\
\hline Yes & 43,452 & 12.4 \\
\hline Had prior biopsy & 16,737 & 5.2 \\
\hline \multicolumn{3}{|l|}{ Highest severity of prior biopsy } \\
\hline Non-proliferative disease & 11,157 & 66.7 \\
\hline Proliferative disease without atypia & 4684 & 28.0 \\
\hline Proliferative disease with atypia & 728 & 4.4 \\
\hline Lobular carcinoma in situ & 168 & 1.0 \\
\hline \multicolumn{3}{|l|}{ BI-RADS density } \\
\hline Almost entirely fat & 32,166 & 10.3 \\
\hline Scattered fibroglandular tissue & 121,751 & 39.1 \\
\hline Heterogeneously dense & 124,730 & 40.0 \\
\hline Extremely dense & 32,909 & 10.6 \\
\hline & 37,399 & \\
\hline \multicolumn{3}{|l|}{ Gail lifetime risk } \\
\hline High $>20 \%$ & 4705 & 1.4 \\
\hline Average $(<20 \%)$ & 343,951 & 98.6 \\
\hline Missing & 299 & \\
\hline
\end{tabular}

BI-RADS: American College of Radiology Breast Imaging Reporting and Data System

women without a prior biopsy. Among biopsied women, the likelihood of MRI increased substantially with increasing degree of high-risk benign lesions. Compared to women whose biopsies had non-proliferative disease, risk of MRI receipt was over seven-fold higher among those with atypia and 33-fold higher among those with LCIS (p-trend $<0.001$ ). Use of screening MRI also increased with BI-RADs breast density category. Women classified as having heterogeneously dense tissue were 1.5 -fold more likely and those with extremely dense tissue were 2.2-fold more likely to be screened with MRI ( $p$-trend < 0.001) than women with scattered fibroglandular tissue. Screening MRI utilization did not differ by age, except that women with a family history were more likely to obtain an MRI if age $<50$ years (RR 11.2) vs. older (RR 6.6) ( $p$-value heterogeneity $<0.0001)$. Women without a firstdegree family history, who have low-to-average risk by family history-based guidelines, received 35.5\% (532/1499; $95 \%$ CI $33.1-37.9 \%$ ) of all MRIs.

The collective influence of breast cancer risk factors on use of supplemental MRI among women without a family history was reflected in the relationship between MRI use and Gail risk score (Table 2). Women with a Gail risk score of 15-19\% lifetime breast cancer risk underwent MRI almost three times more often than women with less than $15 \%$ lifetime risk. 
Table 2 Utilization of Breast Screening Magnetic Resonance Imaging (MRI) Among Women Undergoing Mammography with Less Than 20\% Lifetime Risk of Breast Cancer, as Defined by Family History (Fewer Than Two Affected First-Degree Relatives) $(\mathrm{n}=344,858)$.

\begin{tabular}{|c|c|c|c|c|c|}
\hline Characteristic & Mammograms & MRIs & Relative risk & & 95\% Confidence interval \\
\hline Total & 344,858 & 1242 & & & \\
\hline \multicolumn{6}{|l|}{ All ages: number of first-degree relatives with breast cancer } \\
\hline None & 305,503 & 532 & 1.0 & & \\
\hline One & 39,355 & 710 & 8.1 & 7.2 & 9.1 \\
\hline \multirow{2}{*}{\multicolumn{6}{|c|}{ 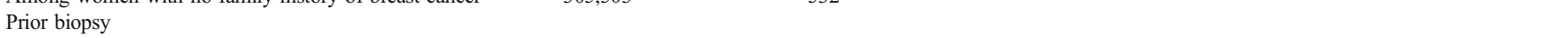 }} \\
\hline & & & & & \\
\hline No & 255,893 & 338 & 1.0 & & \\
\hline Yes & 13,683 & 91 & 4.0 & 3.1 & 5.1 \\
\hline \multicolumn{6}{|l|}{ Prior biopsy result } \\
\hline Non-proliferative disease & 9130 & 35 & 1.0 & & \\
\hline Proliferative disease without atypia & 3867 & 29 & 2.0 & 1.2 & 3.3 \\
\hline Proliferative disease with atypia & 559 & 14 & 7.4 & 3.9 & 14.2 \\
\hline Lobular carcinoma in situ & 127 & 13 & 33.1 & 18.0 & 60.9 \\
\hline \multicolumn{6}{|l|}{ Breast density (BI-RADS) } \\
\hline Almost entirely fat & 28,119 & 15 & 0.4 & 0.2 & 0.6 \\
\hline Scattered fibroglandular tissue & 106,690 & 143 & 1.0 & & \\
\hline Heterogeneously dense & 108,594 & 227 & 1.5 & 1.2 & 1.9 \\
\hline Extremely dense & 28,506 & 93 & 2.2 & 1.7 & 2.8 \\
\hline Missing & 33,324 & 54 & & & \\
\hline \multicolumn{6}{|l|}{ Gail lifetime risk among all women at $<20 \%$ familial risk } \\
\hline$<15 \%$ & 303,984 & 511 & 1.0 & & \\
\hline $15-19 \%$ & 1040 & 8 & 2.8 & 1.4 & 5.6 \\
\hline$>20 \%$ & 216 & 13 & 20.5 & 11.7 & 36.1 \\
\hline Missing & 263 & 0 & & & \\
\hline Age $<50$ years at screening & 144,240 & 707 & & & \\
\hline \multicolumn{6}{|l|}{ Number of first-degree relatives with breast cancer } \\
\hline None & 129,012 & 276 & 1.0 & & \\
\hline One & 15,228 & 431 & 11.2 & 9.6 & 13.0 \\
\hline Among women with no family history of breast cancer & 129,012 & 276 & & & \\
\hline \multicolumn{6}{|l|}{ Prior biopsy } \\
\hline No & 114,977 & 196 & 1.0 & & \\
\hline Yes & 4702 & 45 & 4.1 & 3.0 & 5.7 \\
\hline \multicolumn{6}{|l|}{ Prior biopsy result } \\
\hline Non-proliferative disease & 3130 & 17 & 1.0 & & \\
\hline Proliferative disease without atypia & 1425 & 15 & 2.0 & 1.0 & 4.2 \\
\hline Proliferative disease with atypia & 123 & 7 & 10.2 & 4.2 & 25.0 \\
\hline Lobular carcinoma in situ & 24 & 6 & 38.0 & 16.6 & 87.0 \\
\hline \multicolumn{6}{|l|}{ Breast density (BI-RADS) } \\
\hline Almost entirely fat & 7568 & 8 & 0.62 & 0.3 & 1.3 \\
\hline Scattered fibroglandular tissue & 37,424 & 58 & 1.0 & & \\
\hline Heterogeneously dense & 51,876 & 127 & 1.6 & 1.2 & 2.2 \\
\hline Extremely dense & 17,482 & 55 & 2.1 & 1.4 & 3.0 \\
\hline Missing & 14,662 & 28 & & & \\
\hline \multicolumn{6}{|l|}{ Gail lifetime risk among all women at $<20 \%$ familial risk } \\
\hline$<15 \%$ & 128,077 & 263 & 1.0 & & \\
\hline $15-19 \%$ & 766 & 7 & 3.2 & 1.5 & 6.8 \\
\hline$>20 \%$ & 100 & 6 & 21.2 & 9.5 & 47 \\
\hline Missing & 69 & 0 & & & \\
\hline Age $\geq 50$ years at screening & 200,618 & 535 & & & \\
\hline \multicolumn{6}{|l|}{ Number of first-degree relatives with breast cancer } \\
\hline None & 176,491 & 256 & 1.0 & & \\
\hline One & 24,127 & 279 & 6.6 & 5.6 & 7.9 \\
\hline Among women with no family history of breast cancer & 176,491 & 256 & & & \\
\hline
\end{tabular}


Table 2. (continued)

\begin{tabular}{|c|c|c|c|c|c|}
\hline Characteristic & Mammograms & MRIs & Relative risk & & $95 \%$ Confidence interval \\
\hline \multicolumn{6}{|l|}{ Prior biopsy } \\
\hline No & 140,916 & 142 & 1.0 & & \\
\hline Yes & 8981 & 46 & 3.8 & 2.7 & 5.3 \\
\hline \multicolumn{6}{|l|}{ Prior biopsy result } \\
\hline Nonproliferative disease & 6000 & 18 & 1.0 & & \\
\hline Proliferative disease without atypia & 2442 & 14 & 1.9 & 1.0 & 3.9 \\
\hline Proliferative disease with atypia & 436 & 7 & 5.5 & 2.3 & 13.0 \\
\hline Lobular carcinoma in situ & 103 & 7 & 26.3 & 11.2 & 62.1 \\
\hline \multicolumn{6}{|l|}{ Breast density (BI-RADS) } \\
\hline Almost entirely fat & 20,551 & 7 & 0.3 & 0.1 & 0.5 \\
\hline Scattered fibroglandular tissue & 69,536 & 85 & 1.0 & & \\
\hline Heterogeneously dense & 56,718 & 100 & 1.4 & 1.1 & 1.9 \\
\hline Extremely dense & 11,024 & 38 & 2.6 & 1.8 & 3.9 \\
\hline Missing & 18,662 & 26 & & & \\
\hline \multicolumn{6}{|c|}{ Gail lifetime risk among all women at $<20 \%$ familial risk } \\
\hline$<15 \%$ & 175,907 & 248 & 1.0 & & \\
\hline $15-19 \%$ & 274 & 1 & N.C & & \\
\hline$>20 \%$ & 116 & 7 & 23.1 & 11.0 & 48.7 \\
\hline Missing & 194 & 0 & & & \\
\hline
\end{tabular}

BI-RADS: American College of Radiology Breast Imaging Reporting and Data System MRI, magnetic resonance imaging. N.C. Not calculated because of small cell sizes

Women with more than $20 \%$ lifetime risk were over 20 -fold more likely to receive MRI and generally had breast biopsy results such as atypia. Thus, the Gail model, when applied among women who did not have a first-degree family history, may have influenced MRI use.

In contrast, women with two or more first-degree relatives with breast cancer ( $n=4097 ; 1.2 \%$ of all women; $95 \%$ CI $1.1-$ $1.2 \%$ ) constituted a high-risk group who met the criteria for MRI (20\% or greater lifetime risk) established by professional guidelines (Table 3). In this group, women with additional established breast cancer risk factors were also more likely to undergo screening MRI. Women with a history of breast biopsy were more than two-fold more likely than women without a prior biopsy to be screened (Table 3). The small number of women with high-risk lesions (e.g., LCIS) screened with MRI precluded analysis of trend. Women with heterogeneously dense or extremely dense tissue also were 1.6-1.9 times more likely to undergo supplemental MRI than those with scattered fibroglandular densities. MRI screening was not more common among women with Gail model scores indicating $30 \%$ or greater lifetime risk than women with $20 \%$ or greater lifetime risk. Age at screening did not modify MRI utilization.

Overall, $82.9 \%$ (1242/1499) of all screening MRI examinations were obtained by women for whom MRI was not recommended by professional guidelines because of minimal familial risk (95\% CI $80.8-84.7 \%)$. Thus, only $17.1 \%$ of MRI imaging examinations $(95 \%$ CI $15.3-19.1 \%$ ) would be considered guideline-appropriate. Women with no family history and $15 \%$ or lower lifetime risk as calculated by the Gail model received more than one of three $(34.0 \%$; $511 / 1499 ; 95 \%$ CI $31.7-36.5 \%$ ) screening MRIs.

\section{DISCUSSION}

Our data suggest use of screening MRI is not in concordance with professional society recommendations. We found that at least $6.3 \%$ (257/4097; 95\% CI 5.6-7.1\%) of women at highrisk for breast cancer, defined as two or more first-degree relatives with breast cancer, underwent screening MRI. In contrast, screening MRI was received by at least $0.36 \%$ $(1242 / 344858 ; 95 \%$ CI $0.34-0.38 \%)$ of women who had fewer than two first-degree relatives with breast cancer. While the latter finding might seem of limited consequence, the continued growth of MRI use in a low-risk population poses issues for effective resource utilization; in the context of 39.3 million mammograms annually in the United States, ${ }^{24} 0.36 \%$ implies more than 140,000 MRI screening examinations yearly in low- to average-risk women.

Screening guidelines from professional organizations recommend MRI use only among women deemed at elevated breast cancer risk: high-risk genetic mutation carriers, recipients of chest irradiation prior to age 30 , and women at increased risk as calculated by models that largely depend on family history. Such recommendations are based on the limited evidence available from observational studies, in which women with a high genetic breast cancer risk who received MRI screening had an increased cancer detection rate, ${ }^{5-9}$ and identified cancers were often earlier stage than those detected through screening mammography. ${ }^{5,}{ }^{10}$ However, the absence of data on mortality, the most clinically relevant outcome, together with higher false-positive rates for MRI than mammography, prompted restriction of MRI screening recommendations to women in whom the higher cancer detection rates might 
Table 3 Utilization of Breast Screening Magnetic Resonance Imaging (MRI) Among Women Undergoing Mammography with 20\% or more Lifetime Risk of Breast Cancer, as Defined by Family History (2 or more Affected First-Degree Relatives) $(n=4097)$

\begin{tabular}{|c|c|c|c|c|c|}
\hline \multirow{2}{*}{$\begin{array}{l}\text { Risk factor group } \\
\text { Total }\end{array}$} & \multirow{2}{*}{$\begin{array}{l}\text { Mammography } \\
4097\end{array}$} & \multirow{2}{*}{\begin{tabular}{|l|} 
MRI \\
257
\end{tabular}} & \multirow[t]{2}{*}{ Relative risk } & \multicolumn{2}{|c|}{$\begin{array}{l}\text { 95\% Confidence } \\
\text { interval }\end{array}$} \\
\hline & & & & & \\
\hline \multicolumn{6}{|l|}{ All ages } \\
\hline \multicolumn{6}{|l|}{ Prior biopsy } \\
\hline No & 2898 & 152 & 1.0 & & \\
\hline Yes & 370 & 54 & 2.2 & 1.6 & 2.9 \\
\hline \multicolumn{6}{|l|}{ Prior biopsy result } \\
\hline Non-proliferative disease & 248 & 35 & 1.0 & & \\
\hline Proliferative disease & 95 & 12 & 1.0 & 0.5 & 1.8 \\
\hline Without atypia proliferative disease & 22 & 4 & & N.C & \\
\hline With atypia lobular carcinoma in situ & 5 & 3 & & N.C & \\
\hline \multicolumn{6}{|l|}{ BI-RADS density } \\
\hline Almost entirely fat & 451 & 12 & 0.5 & 0.3 & 0.9 \\
\hline Scattered fibroglandular tissue & 1461 & 71 & 1.0 & & \\
\hline Heterogeneously dense & 1433 & 115 & 1.6 & 1.2 & 2.2 \\
\hline Extremely dense & 383 & 45 & 1.9 & 1.3 & 2.7 \\
\hline Missing & 369 & 14 & & & \\
\hline \multicolumn{6}{|l|}{ Gail model lifetime risk } \\
\hline $20-29 \%$ & 2193 & 139 & 1.0 & & \\
\hline $30 \%+$ & 952 & 93 & 1.1 & 0.8 & 1.4 \\
\hline Missing & 952 & 25 & & & \\
\hline Age $<50$ years at screening & 891 & 107 & & & \\
\hline \multicolumn{6}{|l|}{ Screening prior biopsy } \\
\hline No & 792 & 86 & 1.0 & & \\
\hline Yes & 99 & 21 & 1.6 & 1.0 & 2.3 \\
\hline \multicolumn{6}{|l|}{ Prior biopsy result } \\
\hline Non-proliferative disease & 66 & 13 & 1.0 & & \\
\hline Proliferative disease & 28 & 5 & 1.0 & 0.4 & 2.3 \\
\hline Without atypia proliferative disease & 4 & 2 & N.C & & \\
\hline With atypia lobular carcinoma in situ & 1 & 1 & N.C & & \\
\hline \multicolumn{6}{|l|}{ BI-RADS density } \\
\hline Almost entirely fat & 70 & 6 & 0.8 & 0.4 & 1.7 \\
\hline Scattered fibroglandular tissue & 293 & 29 & 1.0 & & \\
\hline Heterogeneously dense & 392 & 58 & 1.6 & 1.1 & 2.4 \\
\hline Extremely dense & 171 & 21 & 1.4 & 0.8 & 2.2 \\
\hline \multirow{2}{*}{\multicolumn{6}{|c|}{ Gail model lifetime risk }} \\
\hline & & & & & \\
\hline $20-29 \%$ & 401 & 55 & 1.0 & & \\
\hline $30 \%+$ & 411 & 56 & 1.1 & 0.8 & 1.5 \\
\hline Missing & 211 & 9 & & & \\
\hline Age $\geq 50$ years at screening & 2377 & 99 & & & \\
\hline \multicolumn{6}{|l|}{ Prior biopsy } \\
\hline No & 2106 & 66 & 1.0 & & \\
\hline Yes & 271 & 33 & 2.9 & 1.9 & 4.5 \\
\hline \multicolumn{6}{|l|}{ Prior biopsy result } \\
\hline Non-proliferative disease & 182 & 22 & 1.0 & & \\
\hline Proliferative disease & 67 & 7 & 1.0 & 0.4 & 2.2 \\
\hline Without atypia proliferative disease & 18 & 2 & N.C & & \\
\hline With atypia lobular carcinoma in situ & 4 & 2 & N.C & & \\
\hline \multicolumn{6}{|l|}{ BI-RADS density } \\
\hline Almost entirely fat & 381 & 6 & 0.4 & 0.2 & 0.9 \\
\hline Scattered fibroglandular tissue & 1168 & 42 & 1.0 & & \\
\hline Heterogeneously dense & 1041 & 57 & 1.7 & 1.2 & 2.5 \\
\hline
\end{tabular}


Table 3. (continued)

\begin{tabular}{|c|c|c|c|c|c|}
\hline Risk factor group & Mammography & MRI & Relative risk & \multicolumn{2}{|c|}{$\begin{array}{l}\text { 95\% Confidence } \\
\text { interval }\end{array}$} \\
\hline Extremely dense & 212 & 24 & 3.1 & 1.9 & 5.1 \\
\hline Missing & 272 & 8 & & & \\
\hline \multicolumn{6}{|l|}{ Gail model lifetime risk } \\
\hline $20-29 \%$ & 1792 & 84 & 1.0 & & \\
\hline $30 \%+$ & 541 & 37 & 1.3 & 0.9 & 2.0 \\
\hline Missing & 741 & 16 & & & \\
\hline
\end{tabular}

BI-RADS: American College of Radiology Breast Imaging Reporting and Data System. N.C. not calculated because of small cell sizes

offset possible harms, such as the cascade of additional imaging and diagnostic procedures that can accompany a suspicious finding. ${ }^{25}$ Increased cancer detection rates in high-risk populations without definitive proof of survival benefit also suggests possible harm from overdiagnosis. However, for high-risk women, the balance of benefits and harms has been judged to favor annual screening by MRI as one component of a comprehensive cancer surveillance plan. ${ }^{3,26}$

The supplemental MRI screening of women with low-toaverage risk suggests that women and/or their physicians ${ }^{27}$ use MRI for reasons not identified by our study. Concerns about mammography effectiveness or breast density notification laws may lead women at lower risk to seek advanced imaging, while physicians may be influenced by women's expectations, reimbursement mechanisms, risk aversion in a litigious legal climate, and confidence in the clinical effectiveness of MRI. ${ }^{27}$,

${ }^{28}$ Our data suggest that women with atypia, LCIS, or extremely dense breast tissue in particular appear to receive MRI screening more frequently than women without those characteristics. Limited evidence is available regarding the benefits and harms of MRI among intermediate-risk women (above average risk and $<20 \%$ lifetime risk). ${ }^{5,29-32}$ For women at intermediate risk, cancer detection rates by MRI appear similar $^{5}$ or higher ${ }^{29-32}$ than by screening mammography, and recall rates are generally greater. However, even if sensitivity and specificity were equal among intermediate- and high-risk women, the lower cancer incidence in the intermediate-risk population (and thus lower positive predictive value) might preclude routine MRI use. Results of several analyses suggest that MRI screening is currently cost-effective, using standard criteria, only among women with high lifetime breast cancer risk. $^{13,} 14,33,34$ Cancer yield and survival benefit with MRI may have to be high to support annual MRI screening of intermediate risk women.

As the complex balance of potential harms and benefits for screening MRI has been determined to result in a net benefit to women at familial high breast cancer risk, ${ }^{3,26}$ MRI receipt by only a fraction of high-risk women presents an opportunity to improve identification of these women and offer MRI screening. Our data suggest that, after high familial risk was taken into account, other established risk factors for breast cancer played only a modest role in shaping MRI screening decisions.
For instance, women with over $30 \%$ lifetime risk according to the Gail score were no more likely to undergo MRI than women with 20-29\% lifetime risk. Barriers to screening MRI receipt include access, out-of-pocket costs, and ability to tolerate the procedure; these may affect up to $40 \%$ of highrisk women. ${ }^{35}$ These factors, coupled with a lack of recognition of high-risk women who might benefit from additional services, ${ }^{11}$ suggest that if mortality benefits are clearly established, optimizing MRI screening benefits for high-risk women may require reduction of multiple barriers.

Limitations of our study include lack of data on high-risk mutation status, although this should correlate closely with breast cancer family history. We also lack pedigree information on ovarian cancer and on breast and ovarian cancer history in second-degree relatives. As a result, a small proportion of women may have been misclassified as low or average risk. Our analysis is limited to examinations performed in facilities that participate in the BCSC. Some women who received a screening mammogram at a BCSC facility may have received a screening MRI at a non-participating facility. By limiting the analysis to facilities that performed both mammography and MRI, we minimized potential under-ascertainment of MRI use. We are not able to estimate the actual prevalence of MRI use. However, the relative risk of undergoing MRI, comparing risk groups, is unbiased even with incomplete capture of MRIs. ${ }^{23}$ Our study strengths include the availability of screening information from breast imaging facilities in five geographic regions, allowing inferences beyond the specifics of a single practice.

Breast MRI use that is not concordant with guidelines, as documented in this study, poses distinct challenges to effective resource allocation in breast cancer screening. Our data suggest that women with less than $20 \%$ lifetime risk based on family history, but who have high breast density, breast atypia, or LCIS, undergo MRI at many times the rate of other women, although the harms and benefits in this population are uncertain and the cost-benefit ratio may exceed established benchmarks. ${ }^{13,14,33,34}$ Such women may not be at substantially increased risk of cancers missed by mammography (interval cancers) and thus not in need of screening MRI, in part because interval cancer rates are high among women at higher overall breast cancer risk. ${ }^{36}$ In contrast, among women who meet professional guidelines for screening MRI, only a fraction received this service; thus, potential benefits may be missed. 
Our data illustrate the substantial gap between current practice and supplemental MRI breast cancer screening that is both consistent with professional guidelines and the goal of delivery of high-value care. ${ }^{4,37}$ MRI performance measures should be evaluated in non-mutation carriers using validated risk models and consideration of survival benefit to guide MRI utilization.

Acknowledgements: We thank the participating women, mammography facilities, and radiologists for the data they provided for this study.

Corresponding Author: Deirdre A. Hill, Ph.D.; Department of Internal Medicine University of New Mexico School of Medicine, 1 University of New Mexico, MSC 10-5550 87131-0001, Albuquerque, NM, Mexico (e-mail: dahill@salud.unm.edu).

Funding This work was supported by the funds from the National Cancer Institute-funded BCSC (PO1 CA154292, HHSN261201100031C) and the National Cancer Institute (U54 CA163303). The effort of CIL was supported by American Cancer Society Grant 126,947-MRSG-14-160-01-CPHPS. The collection of cancer data used in this study was supported in part by several state public health departments and cancer registries throughout the US. For a full description of these sources, please see: http://www. bcsc-research.org. The content is solely the responsibility of the authors and does not necessarily represent the official views of the National Cancer Institute or the National Institutes of Health. The funders had no role in the design or conduct of the study, in the collection, management, analysis, or interpretation of data, nor in the preparation, review or approval of the manuscript.

\section{Compliance with ethical standards:}

Conflict of interest: None of the authors have identified a conflict of interest in relation to this manuscript.

\section{REFERENCES}

1. Stout NK, Nekhlyudov $\mathbf{L}, \mathbf{L i} \mathbf{L}$, et al. Rapid increase in breast magnetic resonance imaging use: trends from 2000 to 2011. JAMA internal medicine. 2014;174: 114-121.

2. Wernli KJ, DeMartini WB, Ichikawa L, et al. Patterns of breast magnetic resonance imaging use in community practice. JAMA Intern Med. 2014;174: 125-132.

3. Lee CH, Dershaw DD, Kopans D, et al. Breast cancer screening with imaging: recommendations from the Society of Breast Imaging and the ACR on the use of mammography, breast MRI, breast ultrasound, and other technologies for the detection of clinically occult breast cancer. $J$ Am Coll Radiol. 2010;7: 18-27.

4. Wilt TJ, Harris RP, Gaseem A, High Value Care Task Force of the American College of P. Screening for cancer: advice for high-value care from the american college of physicians. Ann Intern Med. 2015;162: 718725.

5. Kriege M, Brekelmans CT, Boetes C, et al. Efficacy of MRI and mammography for breast-cancer screening in women with a familial or genetic predisposition. N Engl J Med. 2004;351: 427-437.

6. Kuhl CK, Schrading $\mathbf{S}$, Leutner $\mathbf{C C}$, et al. Mammography, breast ultrasound, and magnetic resonance imaging for surveillance of women at high familial risk for breast cancer. J Clin Oncol. 2005;23: 8469-8476.

7. Leach MO, Boggis CR, Dixon AK, et al. Screening with magnetic resonance imaging and mammography of a UK population at high familial risk of breast cancer: a prospective multicentre cohort study (MARIBS). Lancet. 2005;365: 1769-1778.

8. Warner E, Plewes DB, Hill KA, et al. Surveillance of BRCA1 and BRCA2 mutation carriers with magnetic resonance imaging, ultrasound, mammography, and clinical breast examination. JAMA. 2004;292: 13171325 .
9. Sardanelli F, Podo F, D'Agnolo G, et al. Multicenter comparative multimodality surveillance of women at genetic-familial high risk for breast cancer (HIBCRIT study): interim results. Radiology. 2007;242: 698-715.

10. Warner E, Hill K, Causer P, et al. Prospective study of breast cancer incidence in women with a BRCA1 or BRCA2 mutation under surveillance with and without magnetic resonance imaging. J Clin Oncol. 2011;29: 1664-1669.

11. Bellcross CA, Leadbetter S, Alford SH, Peipins LA. Prevalence and healthcare actions of women in a large health system with a family history meeting the 2005 USPSTF recommendation for BRCA genetic counseling referral. Cancer Epidemiol Biomarkers Prev. 2013;22: 728-735.

12. Berg WA, Zhang Z, Lehrer $\mathbf{D}$, et al. Detection of breast cancer with addition of annual screening ultrasound or a single screening MRI to mammography in women with elevated breast cancer risk. JAMA. 2012;307: 1394-1404.

13. Saadatmand S, Tilanus-Linthorst MM, Rutgers EJ, et al. Costeffectiveness of screening women with familial risk for breast cancer with magnetic resonance imaging. J Natl Cancer Inst. 2013;105: 1314-1321.

14. Plevritis SK, Kurian AW, Sigal BM, et al. Cost-effectiveness of screening BRCAl/2 mutation carriers with breast magnetic resonance imaging. JAMA. 2006;295: 2374-2384.

15. Tyrer J, Duffy Sw, Cuzick J. A breast cancer prediction model incorporating familial and personal risk factors. Stat Med. 2004;23: 1111-1130.

16. Claus EB, Risch N, Thompson WD. Autosomal dominant inheritance of early-onset breast cancer. Implications for risk prediction. Cancer. 1994;73: 643-651.

17. Antoniou AC, Cunningham AP, Peto J, et al. The BOADICEA model of genetic susceptibility to breast and ovarian cancers: updates and extensions. Br J Cancer. 2008;98: 1457-1466.

18. Ballard-Barbash R, Taplin SH, Yankaskas BC, et al. Breast Cancer Surveillance Consortium: a national mammography screening and outcomes database. AJR Am J Roentgenol. 1997;169: 1001-1008.

19. Santen RJ MR. Benign Breast Disorders. N Engl J Med. 2005;353: 275285.

20. American College of Radiology. The American College of Radiology Breast Imaging Reporting and Data System (BI-RADS). 4th ed. Reston, VA. 2003.

21. Gail MH, Brinton LA, Byar DP, et al. Projecting individualized probabilities of developing breast cancer for white females who are being examined annually. J Natl Cancer Inst. 1989;81: 1879-1886.

22. Zou G. A modified poisson regression approach to prospective studies with binary data. Am J Epidemiol. 2004;159: 702-706.

23. Chubak J, Pocobelli G, Weiss NS. Tradeoffs between accuracy measures for electronic health care data algorithms. J Clin Epidemiol. 2012;65: 343-349 e342.

24. Mammography Quality Standards Act Statistics http://www.fda.gov/ radiation-emittingproducts/mammographyqualitystandardsactandprogram/ucm 113858.htm.Accessed November 21, 2016.

25. Hubbard RA, Kerlikowske K, Flowers CI, Yankaskas BC, Zhu W, Miglioretti DL. Cumulative probability of false-positive recall or biopsy recommendation after 10 years of screening mammography: a cohort study. Ann Intern Med. 2011;155: 481-492.

26. Saslow D, Boetes C, Burke W, et al. American Cancer Society guidelines for breast screening with MRI as an adjunct to mammography. CA: a cancer journal for clinicians. 2007;57: 75-89.

27. Kadivar H, Goff BA, Phillips WR, Andrilla CH, Berg AO, Baldwin LM. Guideline-inconsistent breast cancer screening for women over 50: a vignette-based survey. J Gen Intern Med. 2014;29: 82-89.

28. Hendee WR, Becker GJ, Borgstede JP, et al. Addressing overutilization in medical imaging. Radiology. 2010;257: 240-245.

29. Schwartz T, Cyr A, Margenthaler J. Screening breast magnetic resonance imaging in women with atypia or lobular carcinoma in situ. J Surg Res. 2015; 193: 519-522.

30. Port ER, Park A, Borgen PI, Morris E, Montgomery LL. Results of MRI screening for breast cancer in high-risk patients with LCIS and atypical hyperplasia. Ann Surg Oncol. 2007;14: 1051-1057.

31. Friedlander LC, Roth SO, Gavenonis SC. Results of MR imaging screening for breast cancer in high-risk patients with lobular carcinoma in situ. Radiology. 2011;261: 421-427.

32. Sung JS, Malak SF, Bajaj P, Alis R, Dershaw DD, Morris EA. Screening breast MR imaging in women with a history of lobular carcinoma in situ. Radiology. 2011;261: 414-420. 
33. Moore SG, Shenoy PJ, Fanucchi L, Tumeh JW, Flowers CR. Costeffectiveness of MRI compared to mammography for breast cancer screening in a high risk population. BMC Health Serv Res. 2009;9: 9.

34. Taneja C, Edelsberg J, Weycker D, Guo A, Oster G, Weinreb J. Cost effectiveness of breast cancer screening with contrast-enhanced MRI in high-risk women. J Am Coll Radiol. 2009;6: 171-179.
35. Berg WA, Blume JD, Adams AM, et al. Reasons women at elevated risk of breast cancer refuse breast MR imaging screening: ACRIN 6666. Radiology. 2010;254: 79-87.

36. Kerlikowske $\mathbf{K}$, Zhu W, Tosteson AN, et al. Identifying women with dense breasts at high risk for interval cancer: a cohort study. Ann Intern Med. 2015;162: 673-681. 